\title{
Magnetic dynamo action at low magnetic Prandtl numbers
}

\author{
Leonid M. Malyshkin用 and Stanislav Boldyrev计 \\ ${ }^{1}$ Department of Astronomy $\&$ Astrophysics, University of Chicago, 5640 S. Ellis Ave., Chicago, IL 60637 and \\ ${ }^{2}$ Department of Physics, University of Wisconsin-Madison, 1150 University Ave., Madison, WI 53706
}

(Dated: November 6, 2018)

\begin{abstract}
Amplification of magnetic field due to kinematic turbulent dynamo action is studied in the regime of small magnetic Prandtl numbers. Such a regime is relevant for planets and stars interiors, as well as for liquid metal laboratory experiments. A comprehensive analysis based on the KazantsevKraichnan model is reported, which establishes the dynamo threshold and the dynamo growth rates for varying kinetic helicity of turbulent fluctuations. It is proposed that in contrast with the case of large magnetic Prandtl numbers, the kinematic dynamo action at small magnetic Prandtl numbers is significantly affected by kinetic helicity, and it can be made quite efficient with an appropriate choice of the helicity spectrum.
\end{abstract}

PACS numbers: 47.65.Md, 52.30.-q, 07.55.Db, 96.60.Hv

Introduction.- Turbulent dynamo action is a process of amplification of a weak magnetic field in a conducting turbulent fluid or plasma. It is a plausible mechanism by which cosmic magnetic fields were created [e.g., 19]. Dynamo action has also been studied in laboratory experiments [10 12].

In turbulent fluids possessing velocity fluctuations in a broad range of scales, small-scale fluctuations evolve much faster than large-scale ones. As a result, weak magnetic fields are predominantly amplified at the smallest scales at which the relative motion of the magnetic field lines is not yet significantly affected by magnetic diffusivity. The efficiency of the dynamo action therefore essentially depends on fluid viscosity $\nu$ and magnetic diffusivity $\eta$, whose ratio is characterized by the dimensionless magnetic Prandtl number $P m=\nu / \eta$.

In nature, the $P m$ number is either very large (e.g., interstellar medium) or very small (e.g., planets, stars interiors, liquid metal laboratory experiments). Significant interest has therefore been devoted to the two limiting cases $P m \gg 1$ and $P m \ll 1$. In the first case, the smallscale magnetic field is essentially amplified by smooth, viscous-scale velocity fluctuations, and detailed analytical and numerical treatment is possible, e.g., 13. The opposite case of small $\mathrm{Pm}$ is understood to a much lesser extent, since in this case magnetic fluctuations grow at the scales where the velocity field is non-analytic, [e.g., 14, 15]. It has been established that the dynamo action is less efficient at $P m \ll 1$ compared to its counterpart at $P m \gg 1$, e.g., 11, 14, 16 18]. As a result, numerical and experimental studies of low- $P m$ dynamo action present a significant challenge.

In this Letter we analyze the low-Prandtl number dynamo action with the aid of the kinematic KazantsevKraichnan model [19, 20]. The model allows one to derive the equations for the magnetic field correlation function, which then can be solved numerically for extremely large, practically relevant Reynolds numbers. We found that in contrast with the high- $P m$ dynamo action, low- $P m$ dynamo action is significantly affected by kinetic helicity. Based on our results we propose that the low- $P m$ dynamo action can be made very efficient by ensuring that the velocity fluctuations possess large enough kinetic helicity at the resistive scales.

Formulation of the model. - The evolution of magnetic field $\mathbf{B}(\mathbf{x}, \mathbf{t})$ in magnetohydrodynamics (MHD) is described by the induction equation

$$
\partial_{t} \mathbf{B}=\nabla \times(\mathbf{v} \times \mathbf{B})+\eta \nabla^{2} \mathbf{B}
$$

where $\mathbf{v}(\mathbf{x}, t)$ is the fluid velocity and $\eta$ is the magnetic diffusivity. In general, the velocity fluctuations may possess nonzero kinetic helicity, $H=\int \mathbf{v} \cdot(\nabla \times \mathbf{v}) d^{3} x \neq 0$.

In the Kazantsev-Kraichnan model random turbulent velocity field is assumed to be homogeneous, isotropic, delta-correlated in time, and Gaussian with zero mean, and the covariance tensor

$$
\begin{gathered}
\left\langle v^{i}(\mathbf{x}, t) v^{j}\left(\mathbf{x}^{\prime}, t^{\prime}\right)\right\rangle=\kappa^{i j}\left(\mathbf{x}-\mathbf{x}^{\prime}\right) \delta\left(t-t^{\prime}\right), \\
\kappa^{i j}(\mathbf{x})=\kappa_{N}\left(\delta^{i j}-\hat{x}^{i} \hat{x}^{j}\right)+\kappa_{L} \hat{x}^{i} \hat{x}^{j}+g \epsilon^{i j k} x^{k},
\end{gathered}
$$

where $x=|\mathbf{x}|, \hat{\mathbf{x}} \equiv \mathbf{x} / x,\langle\ldots\rangle$ denotes ensemble average, $\epsilon^{i j k}$ is the unit anti-symmetric pseudo-tensor, and summation over repeated indices is assumed. The first two terms in the right-hand side of Eq. (3) describe the mirror-symmetric, nonhelical part of the turbulence, while the last term describes the helical part. We assume that the velocity is incompressible, resulting in the relation $\kappa_{N}(x)=\kappa_{L}(x)+(x / 2)\left(d \kappa_{L} / d x\right)$. The velocity field is fully specified by the two functions $\kappa_{L}(x)$ and $g(x)$, related to the kinetic energy and kinetic helicity, correspondingly. The Fourier analog of (2, 3) is straightforward:

$$
\begin{gathered}
\left\langle\hat{v}^{i *}(\mathbf{k}, t) \hat{v}^{j}\left(\mathbf{k}, t^{\prime}\right)\right\rangle=\hat{\kappa}^{i j}(\mathbf{k}) \delta\left(t-t^{\prime}\right), \\
\hat{\kappa}^{i j}(\mathbf{k})=F(k)\left(\delta^{i j}-\hat{k}^{i} \hat{k}^{j}\right)+i G(k) \epsilon^{i j l} k^{l},
\end{gathered}
$$

where $\hat{\mathbf{k}}=\mathbf{k} / k$. The functions $F(k)$ and $G(k)$ are related to $\kappa_{L}(x)$ and $g(x)$ by means of the three-dimensional 
Fourier transform [21]. There is a limit to the maximal kinetic helicity due to the Schwarz inequality [6],

$$
|G(k)| \leq F(k) / k .
$$

The correlation function of a homogeneous and isotropic magnetic field can be written similarly to Eq. (3),

$$
\begin{aligned}
\left\langle B^{i}(\mathbf{x}, t) B^{j}(0, t)\right\rangle & =M_{N}\left(\delta^{i j}-\hat{x}^{i} \hat{x}^{j}\right)+M_{L} \hat{x}^{i} \hat{x}^{j} \\
& +K \epsilon^{i j k} x^{k} .
\end{aligned}
$$

Because the magnetic field is divergence-free, we have $M_{N}(x, t)=M_{L}(x, t)+(x / 2)\left(\partial M_{L} / \partial x\right)$. The magnetic field correlator is fully specified by the two functions, $M_{L}(x, t)$ and $K(x, t)$, which correspond to magnetic energy and magnetic helicity respectively. In the kinematic dynamo theory the Lorentz force acting on the magnetized fluid is neglected, and the velocity field (2) is considered to be prescribed.

It turns our that the dynamo problem can be reduced to a quantum mechanical "spinor" form with imaginary time:

$$
-\partial_{t} \psi^{\alpha}(x, t)=\hat{\mathcal{H}}^{\alpha \beta} \psi^{\beta},
$$

where $\alpha=\{1,2\}$ and summation over repeated indices is assumed. The Hamiltonian $\hat{\mathcal{H}}^{\alpha \beta}$ is self-adjoint and it depends on kinetic energy $\kappa_{L}(x)$, kinetic helicity $g(x)$, and magnetic diffusivity $\eta[22]$. The magnetic correlator functions $M_{L}(x, t)$ and $K(x, t)$ can then be expressed in terms of the two components of the function $\psi^{\alpha}(x, t)$. In the case of zero kinetic helicity, $g(x) \equiv 0$, Eq. (8) reduces to the Kazantsev differential equation [19].

Negative eigenvalues of $\hat{\mathcal{H}}^{\alpha \beta},-\lambda<0$, correspond to exponentially growing magnetic fluctuations, $\psi^{\alpha}(x, t) \propto$ $e^{\lambda t}, M_{L}(x, t) \propto e^{\lambda t}$ and $K(x, t) \propto e^{\lambda t}$. Similar to quantum mechanics, in a general case of non-zero kinetic helicity, $g(0) \neq 0$, there are two types of eigenfunctions of Eq. (8) 22, 23]. First, there are unbound (spatially nonlocalized) eigenfunctions which correspond to continuous spectrum $0<\lambda \leq \lambda_{0}$. Here $\lambda_{0}=g^{2}(0) /\left[\kappa_{L}(0)+2 \eta\right]$ is the largest growth rate of an unbound eigenmode. At large $x$ the unbound modes become mixtures of cosine and sine standing waves. Second, there are bound (spatially localized) eigenfunctions that have discrete spectrum $\lambda_{n}>\lambda_{0}, n=1,2, \ldots$. These eigenfunctions decay exponentially fast at large $x$,

$$
\begin{aligned}
M_{L}(x, t) & \propto x^{-2} e^{-k_{r} x} \cos \left[k_{i} x+\phi\right] \\
K(x, t) & \propto x^{-2} e^{-k_{r} x} \sin \left[k_{i} x+\psi\right],
\end{aligned}
$$

where $k_{r}=\sqrt{\lambda_{n}-\lambda_{0}} / \sqrt{\kappa_{L}(0)+2 \eta}$ and $k_{i}=$ $\sqrt{\lambda_{0}} / \sqrt{\kappa_{L}(0)+2 \eta}[22,23]$. Note that the existence of unbound and bound eigenmodes in the helical dynamo problem is similar to existence of "free" and "trapped" quantum particles traveling in a potential well.

As long as the kinetic helicity is non-zero, the unbound eigenmodes always exist in an infinite system. However, real (astro)physical or laboratory systems are limited in size and they may not allow for spatially unbound eigenfunctions. In particular, the unbound solutions should be not relevant if the system size $L$ is smaller than $1 / k_{i} \sim \kappa_{L}(0) / g(0)$. We restrict our consideration to the bound eigenmodes.

We will assume the Kolmogorov scaling of velocity fluctuations, $\left\langle|\hat{v}(\mathbf{k}, t)|^{2}\right\rangle \sim v_{0}^{2} l_{0}^{-2 / 3} k^{-11 / 3}$, with the corresponding eddy turnover time $\tau(k) \sim v_{0}^{-1} l_{0}^{1 / 3} k^{-2 / 3}[24]$. Here $v_{0}$ and $l_{0}$ are the velocity and the scale associated with the largest eddies. The final equation (8) in the Kazantsev-Kraichnan model involves only the integral of the velocity correlation function over time, that is, the turbulent diffusivity, which in Kolmogorov turbulence scales as $\left\langle|\hat{v}(\mathbf{k}, t)|^{2}\right\rangle \tau(k) \sim v_{0} l_{0}^{-1 / 3} k^{-13 / 3}$. By requiring that the Kazantsev-Kraichnan model have the same scaling of turbulent diffusivity, we obtain $\int\left\langle\hat{v}^{i *}(\mathbf{k}, t) \hat{v}^{i}\left(\mathbf{k}, t^{\prime}\right)\right\rangle d t=\hat{\kappa}^{i i}(\mathbf{k}) \sim v_{0} l_{0}^{-1 / 3} k^{-13 / 3}$, and, therefore, $F(k)=\hat{\kappa}^{i i}(\mathbf{k}) / 2 \sim v_{0} l_{0}^{-1 / 3} k^{-13 / 3}$. Without loss of generality we assume $l_{0} \sim 1, v_{0} \sim 1$, and we also choose

$$
F(k)=C k^{-13 / 3}, \quad G(k)=-h F(k) / k
$$

for $2 \leq k \leq k_{\max }$, and $F(k)=G(k)=0$ otherwise. Here $C=(1 / 2 \pi) /\left(2^{-4 / 3}-k_{\max }^{-4 / 3}\right) \sim 1$ is a normalization coefficient chosen such that $\kappa_{L}(0)=1$; note that $\kappa_{L}(0) \sim l_{0} v_{0}$ is the turbulent diffusivity. The maximal wavenumber is $k_{\max } \approx 2\left[\kappa_{L}(0) / \nu\right]^{3 / 4}=2 \nu^{-3 / 4}$, where $\nu$ is the kinematic viscosity. For convenience, we specify $k_{\max }$, and then define the effective viscosity as $\nu=\kappa_{L}(0)\left(2 / k_{\max }\right)^{4 / 3}=\left(2 / k_{\max }\right)^{4 / 3}$. The simple choice of $G(k)$ in Eq. (10), with $-1 \leq h \leq 1$ due to the realizability condition (6), assures that kinetic helicity is present on all scales (we study the effect of helicity on different scales below). The helicity is maximal when $|h|=1$. We define the Reynolds number as $R e=\kappa_{L}(0) / \nu=1 / \nu$, the magnetic Reynolds number as $R m=\kappa_{L}(0) / \eta=1 / \eta$, and the magnetic Prandtl number as $P m=R m / R e=\nu / \eta$.

For exponentially growing magnetic field, $\psi^{\alpha}(x, t) \propto e^{\lambda t}$, Eq. (8) turns into a system of two ordinary differential equations with variable coefficients. We integrate this system numerically by the fourthorder Runge-Kutta method on a nonuniform numerical grid, as described in [23]. As a result, we find the growth rates and the eigenfunctions of all the bound and unbound modes. The bound eigenfunctions decline exponentially at infinity, therefore, they are set to zero at the right boundary of our computational interval.

Dynamo growth rate.-As follows from the phenomenological discussion in the introduction, weak magnetic field is most efficiently amplified by turbulent eddies at the resistive scale. Using the Kolmogorov scaling $v_{l} \sim l^{1 / 3}$, we find from Eq. (1) that the resistive scale is $l_{\eta} \sim \eta^{3 / 4}$, and the eddy turnover time at this 


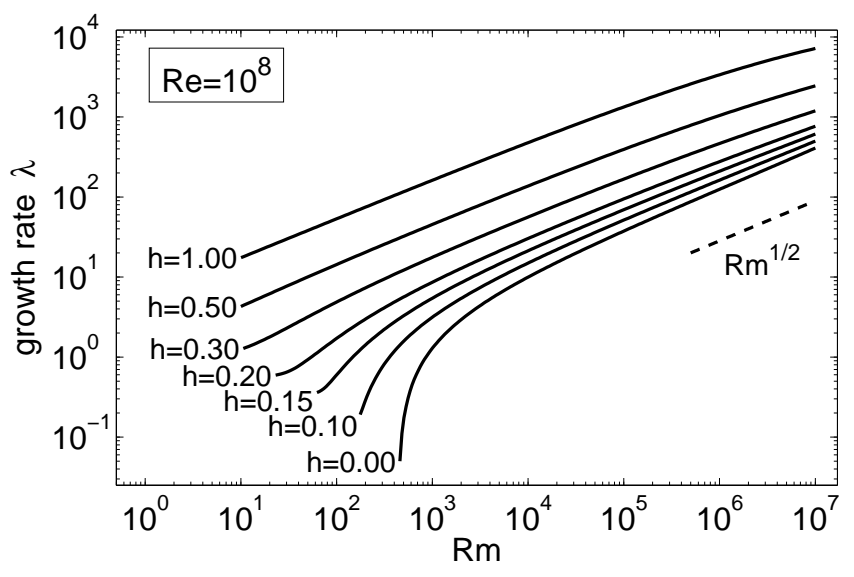

FIG. 1: Scaling of the dynamo growth rate vs the magnetic Reynolds number $R m$, obtained for various levels of kinetic helicity $h$.

scale is $\tau_{\eta} \sim l_{\eta} / v_{\eta} \sim \eta^{1 / 2}$. The dynamo growth rate should therefore scale as $\lambda \sim 1 / \tau_{\eta} \sim R m^{1 / 2}$, see, e.g., [14, 16, 17].

This result is hard to check in low- $P m$ direct numerical simulations, since the range of $R m$ numbers available in such simulations is significantly limited (see, however, [25], where some indication of such scaling was found in the case $P m \sim 1)$. Here we check this scaling in the Kazantsev-Kraichnan model. We solve the model for $R e \sim 10^{8}$, and for the magnetic Reynolds numbers up to $R m \sim 10^{7}$. The results are presented in Fig. (11).

The phenomenological scaling $\lambda \sim R m^{1 / 2}$ is clearly seen in this plot for the zero helicity case $h=0$ [27]. Moreover, we find that the scaling is very close to $\lambda \sim R m^{1 / 2}$ even when kinetic helicity is non-zero. We also observe that for moderate helicities the asymptotic scaling $\lambda \sim R m^{1 / 2}$ is established only at very large $R m$ numbers. We therefore expect that observation of such a scaling will present significant challenge for numerical simulations and for laboratory liquid-metal experiments. On a more optimistic note, we observe that kinetic helicity facilitates dynamo action; this effect will be more evident in our discussion of the dynamo threshold in the next section.

Dynamo threshold.-A crucial question of whether the dynamo action can be observed in a particular system is related to the question of dynamo threshold. We address this question by solving the model for the system size $L=1 / 0.3$ in units of the largest turbulent eddy size $l_{0} \sim 1$. We consider the Reynolds numbers ranging from $R e \approx 1$ to $R e \approx 10^{6}$, corresponding to the maximal wavenumber ranging from $k_{\max }=3$ to $k_{\max }=7 \times 10^{4}$. For each $R e$ (and the corresponding $k_{\max }=2 R e^{3 / 4}$ ) we find the critical magnetic Reynolds number $R m_{c}$ for which there is at least one growing bound mode with $k_{r}>1 / L$. The resulting $R m_{c}$ values are shown for dif-

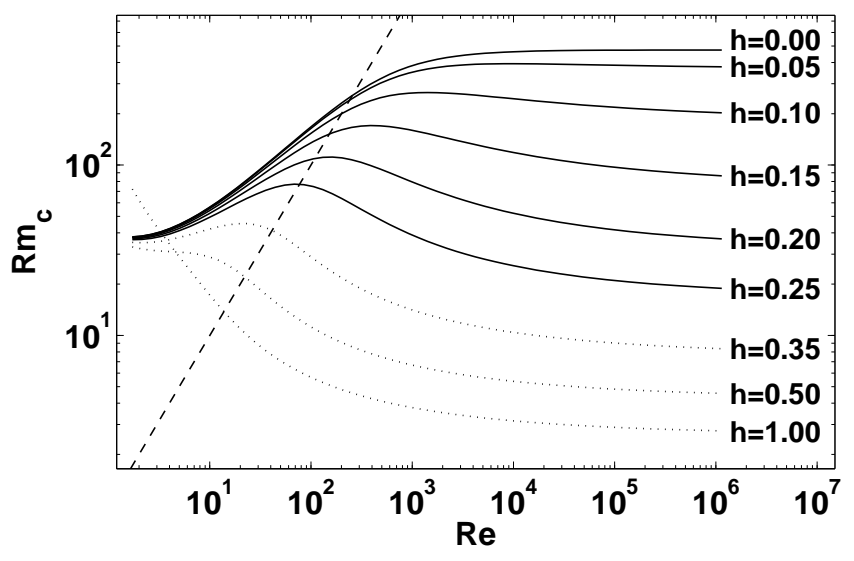

FIG. 2: Stability curves for different values of kinetic helicity $h$ for the box size $L=1 / 0.3$. The curves correspond to the bound modes, that is, the modes with $k_{r}>1 / L$. For $h>\kappa(0) / g(0) \sim 0.3$, the unbound modes can also be excited in the box. In these cases, the presented $R m_{c}$ for the bound modes (shown by dotted lines) give the upper boundary for the true $R m_{c}$. The dashed line corresponds to $P m=1$.

ferent values of kinetic helicity $h$ in Figure 2 The dashed line corresponds to $R m=R e$, that is, $P m=1$. We now make the following two important observations.

First, in the case when kinetic helicity is zero, $h=0$, the critical magnetic Reynolds numbers $R m_{c}$ are considerably higher in low- $P m$ turbulence, than in high- $P m$ turbulence. This result is in agreement with previous analytical and numerical studies of low- $P m$ dynamo in various geometries [14, 16 18]. The situation changes drastically when $h \neq 0$. We find that the critical $R m$ in low- $P m$ turbulence is very sensitive to the amount of kinetic helicity, while it is practically independent of kinetic helicity in high- $\mathrm{Pm}$ turbulence. We will analyze this important property in more detail below.

The second observation is that the stability curve $R m_{c}(R e)$ ceases to be monotone as kinetic helicity increases. The curve peaks around $R m \sim R e$, before it declines and eventually flattens at $R e \gg R m$. This overshooting effect seems to be a robust feature of recent numerical simulations in periodic boxes and in spherical geometry [16 18], although its origin has not been understood. Here we propose that the overshooting is a consequence of kinetic helicity of the velocity fluctuations. Even if kinetic helicity is zero overall (as, e.g., is [16]), it can possess spatial variations, which can affect small scale dynamo action.

Role of kinetic helicity.- - In order to elucidate the role of kinetic helicity in low- $P m$ dynamo, we study at what scales the influence of kinetic helicity is most significant. We set $R e=10^{8}$, and consider several magnetic Reynolds numbers well above the dynamo threshold, where the growth rates are given by Fig. (1) in the fully helical case $(h=1)$. However, instead of considering the fully 


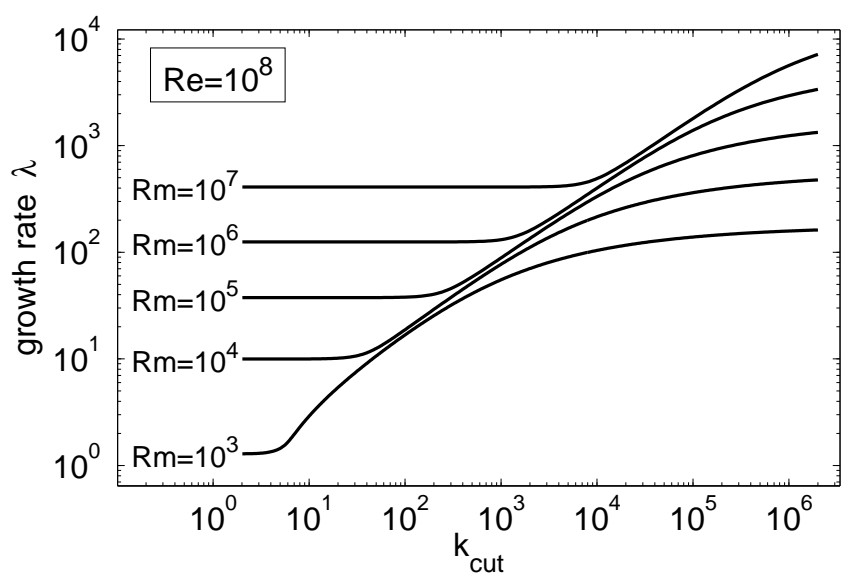

FIG. 3: Dynamo growth rates for varying spectrum of magnetic helicity: $G(k)=0$ at $k>k_{\text {cut }}$ and $G(k)=-F(k) / k$ at $k \leq k_{\text {cut }}$.

helical case, we now modify the helicity spectrum $G(k)$ in Eq. (10) as follows. We set $G(k)=0$ at large wavenumbers $k>k_{\text {cut }}$ and keep $G(k)=-F(k) / k$ at $k \leq k_{\text {cut }}$. We then study how the dynamo growth rate changes as we change the cutoff $k_{\text {cut }}$. The result is presented in Fig. (3).

We observe that the presence of large-scale kinetic helicity (small $k_{\text {cut }}$ ) does not affect the growth rate. Varying kinetic helicity at very small scales (large $k_{\text {cut }}$ ) does not affect the growth rate either. Since the only characteristic scale present in the kinetic inertial interval is the resistive scale, $k_{\eta} \sim R m^{3 / 4}$, we suggest that kinetic helicity significantly affects the dynamo action only when it is present at the resistive scales of magnetic fluctuations. This statement is physically reasonable since, as was explained earlier, it is the resistive scales where the growing magnetic field is concentrated in the kinematic dynamo action. The scaling of the critical cut-off number $k_{\text {cut }} \sim R \mathrm{~m}^{3 / 4}$ is indeed consistent with the behavior observed in Fig. (3).

Conclusions.-We studied kinematic dynamo action in low-Pm turbulence in the framework of the solvable Kazantsev-Kraichnan model [19, 20]. Based on the obtained results we proposed the explanations for somewhat puzzling results of recent direct numerical simulations of low- $P m$ dynamo action, concerning the dynamo threshold, and the scaling of the dynamo growth rate with the magnetic Reynolds number. We also proposed that the low- $P m$ turbulent dynamo action can be made very efficient by ensuring that the velocity fluctuations possess large enough kinetic helicity at the resistive scales.

We thank Fausto Cattaneo and Joanne Mason for helpful discussions. This work was supported by the NSF Center for Magnetic Self-Organization at the University of Chicago under the NSF award PHY-0821899 (LMM), and by the US DoE grant DE-FG02-07ER54932 and the US DoE Early Career Award de-sc0003888 (SB).

* Electronic address: leonmal@uchicago.edu

$\dagger$ Electronic address: boldyrev@wisc.edu

[1] R. M. Kulsrud, R. M., Plasma Physics for Astrophysics (Princeton Univ. Press, Princeton, NJ, 2005).

[2] A. Brandenburg and K. Subramanian, Phys. Rep. 417, 1 (2005).

[3] E. Blackman and H. Ji, Mon. N. R. Astron. Soc. 369, 1837 (2006).

[4] R. M. Kulsrud and E. G. Zweibel, Rep. Prog. Phys. 71, 046901 (2008).

[5] D. Lynden-Bell (ed.), Cosmical Magnetism (Kluwer, Dordrecht, Holland, 1994).

[6] H. K. Moffatt, Magnetic Field Generation in Electrically Conducting Fluids (Cambridge University Press, Cambridge, England, 1978).

[7] E. N. Parker, Cosmical Magnetic Fields (Clarendon Press, Oxford, England, 1979).

[8] A. A. Schekochihin and S. C. Cowley, Astron. Nachr. 327, 599 (2006).

[9] E. G. Zweibel and C. Heiles, Nature 385, 131 (1997).

[10] R. Monchaux et al., Phys. Rev. Lett. 98, 044502 (2007).

[11] E. J. Spence et al., Phys. Rev. Lett. 96, 055002 (2006).

[12] E. J. Spence, K. Reuter and C. B. Forest, Astrophys. J. 700, 470 (2009).

[13] A. A. Schekochihin, S. Boldyrev and R. Kulsrud, Astrophys. J. 567, 828 (2002).

[14] S. Boldyrev and F. Cattaneo, Phys. Rev. Lett. 92, 144501 (2004).

[15] G. L. Eyink and A. F. Neto, New J. Phys. 12, 023021 (2010).

[16] A. B. Iskakov et al., Phys. Rev. Lett. 98, 208501 (2007).

[17] K. Reuter, "Numerical investigation of turbulent dynamo excitation in a spherical MHD system", Ph. D. Thesis (Max-Planck-Institut für Plasmaphysik, Garching, 2010).

[18] Y. Ponty et al., Phys. Rev. Lett. 94, 164502 (2005).

[19] A. P. Kazantsev, JETP 26, 1031 (1968).

[20] R. H. Kraichnan, Phys. Fluids 11, 945 (1968).

[21] A. S. Monin and A. M. Yaglom, Statistical Fluid Mechanics (MIT Press, Cambridge, MA, 1971).

[22] S. Boldyrev, F. Cattaneo and R. Rosner, Phys. Rev. Lett. 95, 255001 (2005).

[23] L. Malyshkin and S. Boldyrev, Astrophys. J. Lett. 671, L185 (2007).

[24] U. Frisch, Turbulence (Cambridge Univ. Press., Cambridge, England, 1995).

[25] N. E. L. Haugen, A. Brandenburg and W. Dobler, Phys. Rev. E 70, 016308 (2004).

[26] I. Rogachevskii and N. Kleeorin, Phys. Rev. E 56, 417 (1997).

[27] An earlier attempt to derive such a scaling from the Kazantsev-Kraichnan model produced a different result $\lambda \sim \log (R m)$ [26]. The discrepancy is a consequence of an incorrect asymptotic matching procedure employed in [26] for establishing the dynamo threshold and calculating the dynamo growth rate. 